\title{
Development of a Portable Muon Witness System
}

\author{
E Aguayo JL Orrell \\ RT Kouzes
}

January 2011

Pacific Northwest

NATIONAL LABORATORY

Proudly Operated by Battelle Since 1965 


\title{
DISCLAIMER
}

This report was prepared as an account of work sponsored by an agency of the United States Government. Neither the United States Government nor any agency thereof, nor Battelle Memorial Institute, nor any of their employees, makes any warranty, express or implied, or assumes any legal liability or responsibility for the accuracy, completeness, or usefulness of any information, apparatus, product, or process disclosed, or represents that its use would not infringe privately owned rights. Reference herein to any specific commercial product, process, or service by trade name, trademark, manufacturer, or otherwise does not necessarily constitute or imply its endorsement, recommendation, or favoring by the United States Government or any agency thereof, or Battelle Memorial Institute. The views and opinions of authors expressed herein do not necessarily state or reflect those of the United States Government or any agency thereof.

\author{
PACIFIC NORTHWEST NATIONAL LABORATORY \\ operated by \\ BATTELLE \\ for the \\ UNITED STATES DEPARTMENT OF ENERGY \\ under Contract DE-AC05-76RL01830
}

Printed in the United States of America

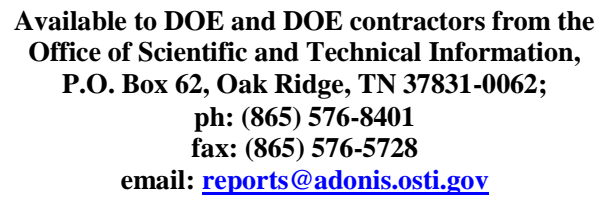

Available to the public from the National Technical Information Service, U.S. Department of Commerce, 5285 Port Royal Rd., Springfield, VA 22161 ph: (800) 553-6847 fax: (703) 605-6900

email: orders@ntis.fedworld.gov online ordering: http://www.ntis.gov/ordering.htm 
PNNL-20194

\title{
Development of a Portable Muon Witness System
}

\author{
E Aguayo JL Orrell \\ RT Kouzes
}

January 2011

Prepared for

the U.S. Department of Energy

under Contract DE-AC05-76RL01830

Pacific Northwest National Laboratory

Richland, Washington 99352 


\begin{abstract}
Since understanding and quantifying cosmic ray induced radioactive backgrounds in copper and germanium are important to the MAJORANA DEMONSTRATOR, methods are needed for monitoring the levels of such backgrounds produced in materials being transported and processed for the experiment. This report focuses on work conducted at Pacific Northwest National Laboratory to develop a muon witness system as a one way of monitoring induced activities. The operational goal of this apparatus is to characterize cosmic ray exposure of materials. The cosmic ray flux at the Earth's surface is composed of several types of particles, including neutrons, muons, gamma rays and protons. These particles induce nuclear reactions, generating isotopes that contribute to the radiological background. Underground, the main mechanism of activation is by muon produced spallation neutrons since the hadron component of cosmic rays is removed at depths greater than a few tens of meters. This is a sub-dominant contributor above ground, but muons become predominant in underground experiments. For low-background experiments cosmogenic production of certain isotopes, such as ${ }^{68} \mathrm{Ge}$ and ${ }^{60} \mathrm{Co}$, must be accounted for in the background budgets. Muons act as minimum ionizing particles, depositing a fixed amount of energy per unit length in a material, and have a very high penetrating power. Using muon flux measurements as a "witness" for the hadron flux, the cosmogenic induced activity can be quantified by correlating the measured muon flux and known hadronic production rates. A publicly available coincident muon cosmic ray detector design, the Berkeley Lab Cosmic Ray Detector (BLCRD), assembled by Juniata College, is evaluated in this work. The performance of the prototype is characterized by assessing its muon flux measurements. This evaluation is done by comparing data taken in identical scenarios with other cosmic ray telescopes. The prototype is made of two plastic scintillator paddles with associated electronics to measure energy depositions in coincidence in the two paddles. For this particular application of the prototype, the measurements performed concentrated on a broad investigation of the dependence of the muon flux on depth underground. These tests were conducted inside at Building 3420/1307 and underground at Building 3425 at the Pacific Northwest National Laboratory. The second half of this report analyzes modifications to the electronics of the BLCRD to make this detector portable. Among other modifications, a battery powered version of these electronics is proposed for the final Muon Witness design.
\end{abstract}




\section{Contents}

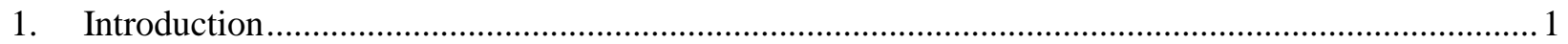

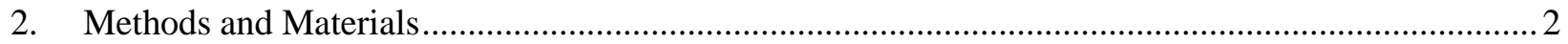

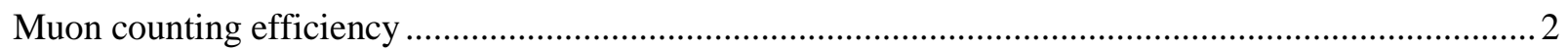

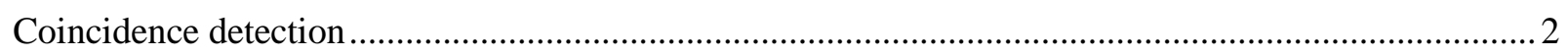

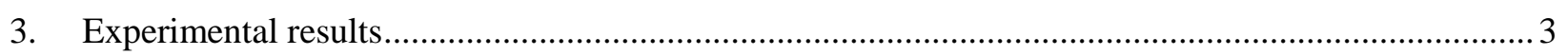

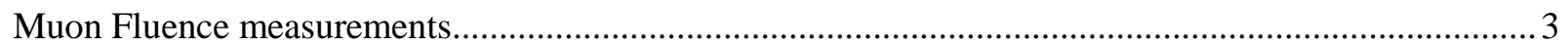

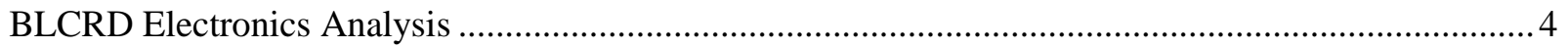

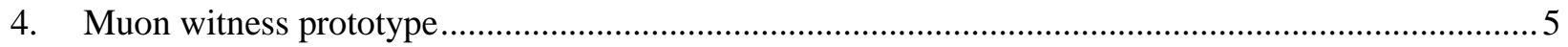

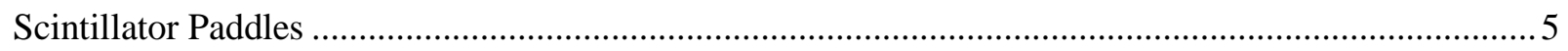

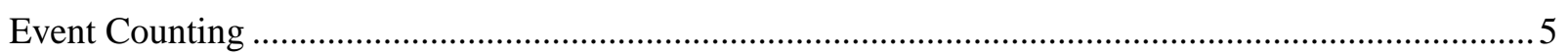

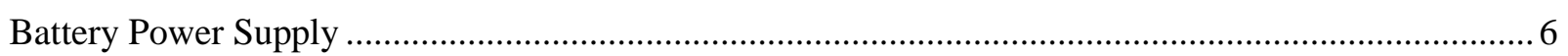

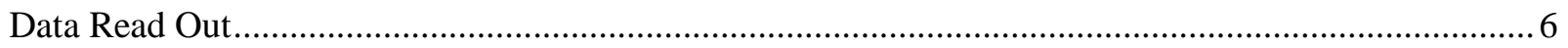

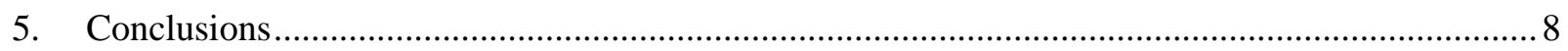

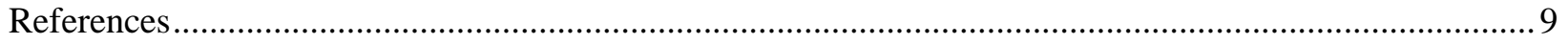




\section{Figures}

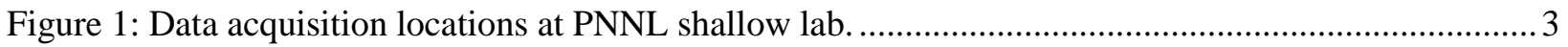

Figure 2: BLCRD electronics board schematics. Taken from [4] …..................................................... 6

Figure 3: Proposed microcontroller board for the Muon Witness .......................................................... 7 


\section{Introduction}

Observing the neutrinoless double beta decay (DBD) in enriched germanium with a half-life in the order of $10^{25}$ years requires a long measurement time and a large amount of source material. Experiments such as the MAJORANA DEMONSTRATOR [1] will be a probe for events with such a long half-life. The background level, and therefore the shielding of the experiment, will play a pivotal role in the success of the observation of the neutrinoless DBD. Cosmic ray witnessing will be used as a tool for the quantification of the activation that is takes place in the germanium and copper while it is being processed and transported on the surface. The induced activity in the detector materials can be calculated using the production rate for the isotope of interest. This production rate is given by equation (1).

$$
\mathrm{Rx}=\Phi(\mathrm{E}) * \sigma_{x}(E) * \rho_{A}=\mathrm{Px} * \rho_{K g}
$$

Where:

- $\quad R_{x}$ is the number of reactions of type $\mathrm{x}$, units: [1/time * volume]

- $\Phi(\mathrm{E})$ is the neutron and protons flux, units: [1/area * time]

- $\sigma_{x}(E)$ is the microscopic cross section for reaction $x$, units: [area]

- $\rho_{\mathrm{A}}$ is the density of atoms in the target in units of [1/volume]

- $\quad \rho_{\mathrm{Kg}}$ is the density of mass in the target in units of [mass/volume]

- Px is the production rate [1/time *mass]

There are two energy dependant terms in the calculation of the production rate. First is the microscopic cross-section for the reaction. The second one is the neutron and proton flux. The cross sections for production rate calculations are generated using nuclear reaction codes [9] and/or experimental reactor nuclear data libraries [8]. The neutron flux from cosmic rays has been studied in detail [6,7]. The neutron flux and the muon flux are correlated on the surface. Using measurements of the muon flux, the neutron flux can be inferred and used to calculate production rates at different locations. The muon witness detector will function as a counter that has an understood relationship between its count rate and a model of the cosmic ray flux in for a given latitude, elevation, and shielding configuration [13]. The evaluation of the suitability of using the BLCRD design as the basis for a portable muon witness detector is the objective of this work. This architecture features a low sensitivity to protons and neutrons, which is desired to easily scale from witness muon counter to total cosmic ray flux. 


\section{Methods and Materials}

An implementation of the BLCRD [3] was carried out by J. Borgardt et al. at Juniata College. This prototype was borrowed by PNNL to evaluate the possibilities of such a detector for the muon witness implementation. The BLCRD was used to acquire the data presented in this report. The prototype features two paddles of scintillator plastic (area of $228.75 \mathrm{~cm} 2$ and $1 \mathrm{~cm}$ thick) with a fixed separation of $13 \mathrm{~cm}$. The electronics board of the prototype features coincidence detection capabilities. The prototype has a set threshold and a 3 digit LED display to read-out the number of counts.

\section{Muon counting efficiency}

Muons travel at velocities approaching the speed of light. These particles act as minimum ionizing particles depositing a fixed amount of energy, $2 \mathrm{MeV} / \mathrm{cm}$ [10]. A broad muon peak at about $2 \mathrm{MeV}$ is expected for the scintillator material thickness of the BLCRD. A substantial gamma contribution to the number of coincidence counts is expected, since the background gamma ray continuum usually tails to a maximum energy of $3 \mathrm{MeV}$. The BLCRD prototype does not feature a spectrometric analysis of the deposited energy in it. The overlap between highenergy gamma rays and muons cannot be measured without adding an adjustable threshold. A way to test the muon efficiency of the BLCRD qualitatively is to use a material layer acting as a gamma-ray shield between the paddles, so that gamma rays are attenuated.

\section{Coincidence detection}

The time performance of the BLCRD is mandated by the decay time of the scintillator plastic used. The coincidence window is equal to the amount of time that the scintillator is emitting light after the energy deposition takes place. For a typical plastic scintillator with a decay time of 100 ns, the coincidence time window should be equal to this time. That means that the maximum detection rate is $10 \mathrm{MHz}$. The output of the scintillator may have some tailing depending on the time constant of the electrical components of the electronic coincidence detection system. 


\section{Experimental results}

\section{Muon Fluence measurements}

In order to test the capabilities of the BLCRD architecture to measure the muon flux, several sets of measurements were taken in the shallow lab, and at an above ground lab, at PNNL. These spots have been previously used as muon flux measurement locations [2, 3]. The results obtained in these measurements can be compared with previous experiments at the same locations. Figure 1 shows the floor plan of the shallow lab. The red dots show where the measurements in Table 1 were taken. The first spot is $1.83 \mathrm{~m}$ away from the elevator shaft. About $5.49 \mathrm{~m}$ from the elevator shaft, right in front of the bathroom, another set of measurements were taken. Inside the bathroom another set of measurements were taken, approximately $7.32 \mathrm{~m}$ from the elevator shaft. The last set of measurements was taken in the very back corner of the shallow lab.

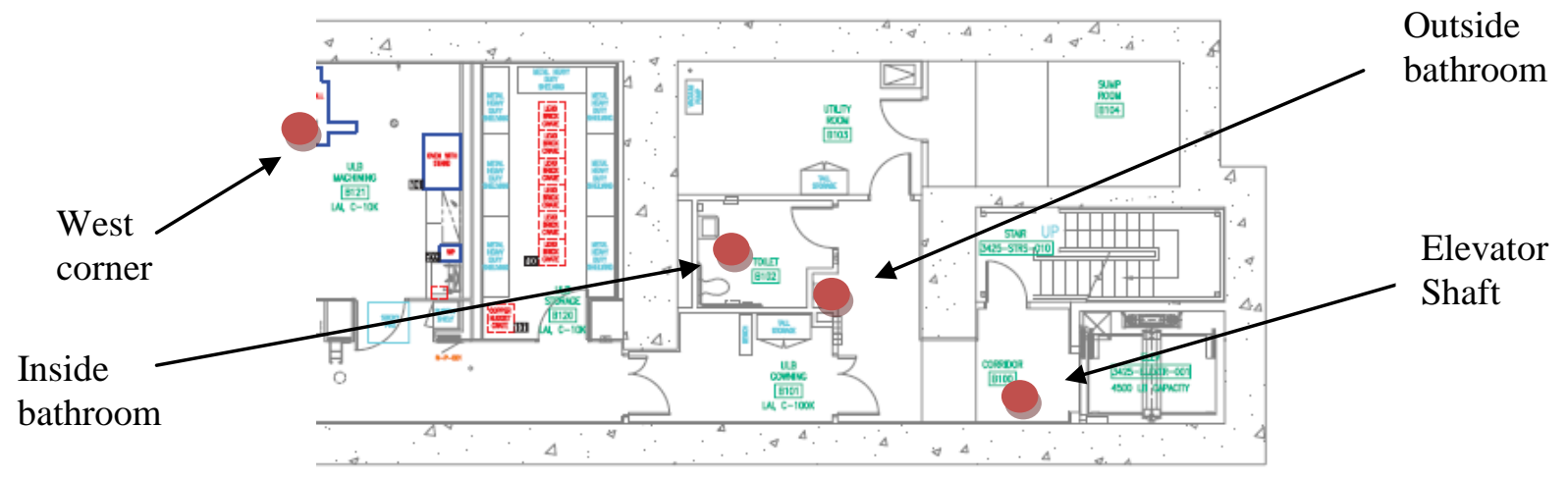

Figure 1: Data acquisition locations at PNNL shallow lab.

\begin{tabular}{|c|c|c|c|c|c|}
\hline & West Corner & $\begin{array}{c}\text { Inside } \\
\text { Bathroom }\end{array}$ & Elevator Shaft & $\begin{array}{c}\text { Outside } \\
\text { Bathroom }\end{array}$ & Lab 1307 \\
\hline 1 & 261 & 371 & 464 & 354 & 877 \\
\hline 2 & 293 & 358 & 459 & 314 & 907 \\
\hline 3 & 265 & 371 & 487 & 348 & 914 \\
\hline 4 & 279 & & & 363 & 945 \\
\hline 5 & & & & 305 & 892 \\
\hline 6 & & & & 328 & 873 \\
\hline 7 & & & & 384 & 886 \\
\hline Total & $\mathbf{2 7 5} \pm \mathbf{1 2}$ & $\mathbf{3 6 7} \pm \mathbf{6}$ & $\mathbf{4 7 0} \pm \mathbf{1 1}$ & $\mathbf{3 4 2} \pm \mathbf{2 3}$ & $\mathbf{9 0 0} \pm \mathbf{2 0}$ \\
\hline
\end{tabular}

Table 1: BLCRD coincidence counts in different locations

Data in Table 1 was collected over 10 minute intervals, and so was scaled to 20 -minute runs for comparative purposes in Table 2. Using the data presented in Table 2, the results from [2, 3] can be compared with those obtained using the BLCRD. In the results reported in [2], the total muon count is derived using measurements from different spots in the lab. The results reported in [3] are for a particular spot in the shallow lab, right in front of the bathroom. 


\begin{tabular}{|l|l|l|l|l|l|}
\hline & Detector used & $\begin{array}{l}\text { Collection } \\
\text { times }\end{array}$ & $\begin{array}{l}\text { Counts at } \\
\text { surface }\end{array}$ & $\begin{array}{l}\text { Counts in } \\
\text { Deep Lab }\end{array}$ & $\begin{array}{l}\text { Ratio } \\
\text { Deep Lab } \\
\text { to surface }\end{array}$ \\
\hline August 2009 [2] & 1-4"x4"x16" NaI(Tl) log & $20 \mathrm{~min}$ & 3795 & 532 & 0.140 \\
\hline July 2010 [3] & $\begin{array}{l}2-5 \mathrm{~cm} 40 \mathrm{~cm} x 120 \mathrm{~cm} \text { PVT } \\
\text { panels in coincidence }\end{array}$ & $\begin{array}{l}3 \mathrm{~min} \\
(\text { surface) or } \\
10 \mathrm{~min} \\
\text { (depth) }\end{array}$ & 43220 & 4562 & 0.106 \\
\hline $\begin{array}{l}\text { January 2011 } \\
\text { (This work) }\end{array}$ & $\begin{array}{l}\text { 2-1.25cmx14.5cmx 10.5cm } \\
\text { Plastic panels in } \\
\text { coincidence }\end{array}$ & $10 \mathrm{~min}$ & $1798 \pm 100$ & $727 \pm 100$ & 0.40 \\
\hline
\end{tabular}

Table 2: Summary of data comparing muon fluence at surface to that in the 3425 shallow lab

From the results of Table 2 a significant difference between the two previous measurements and this measurement can be observed. This difference was attributed to a large number of false coincidences in the BLCRD. In order to test this assertion, an extra set of measurements was carried out using a material in between the scintillator paddles to attenuate gamma rays that could be the cause for the false coincidences. A set of measurements was taken to test the gamma ray coincidence ratio in the total count observed by the BLCRD. A reduction in the measured flux of $10 \%$ at the surface (30\% underground) using a 2.5 in thick paper block in between the paddles was observed. The total contribution of these gamma rays is much more prominent in underground measurements, where the muon flux is attenuated, but the surrounding concrete, where these gamma rays are generated, is much closer. This brings the ratio down from 0.4 to about 0.2 , indicating that gamma ray coincidences may well be the source of the difference between the previous and current measurements. Improvements will be implemented in the next detector to reduce these problems.

\section{BLCRD Electronics Analysis}

A series of measurements to characterize the power consumption of the BLCRD electronics were taken. A regulated power source with an amp meter was utilized during this experimentation. All measurements were taken with a set voltage output of $12 \mathrm{~V}$ from the regulated source. Table 3 shows the results of experimenting with the PMT connections and using the power source amp meter to derive the power consumption. The board draws $200 \mathrm{~mA}$ when in a ready state with the two paddles disconnected. By alternating the paddle connections and operating the detector we concluded that each paddle uses $12 \mathrm{~mA}$. Therefore the main current draw is by the electronics board. In particular, this board uses three large LEDs (PN67-1485-ND) with a reported [5] 80 $\mathrm{mA}$ maximum current consumption. From this data, one can infer that the power draw of the instrument is approximately $0.2 \mathrm{~W}$ if the LEDs are removed.

\begin{tabular}{|c|c|c|c|c|}
\hline $\begin{array}{c}\text { BLCRD } \\
\text { connections }\end{array}$ & PMT1 + PMT2 & PMT1 ONLY & PMT2 ONLY & NONE \\
\hline $\begin{array}{c}\text { Power } \\
\text { Consumption (W) }\end{array}$ & 2.8 & 2.7 & 2.7 & 2.5 \\
\hline
\end{tabular}

Table 3: Power consumption measurements of the prototype 


\section{Muon witness prototype}

This section presents the proposed design features for a portable muon witness prototype based on the results obtained with the BLCRD.

\section{Scintillator Paddles}

The energy deposited in the scintillator paddle by muons is directly proportional to the scintillator thickness. Using a thicker scintillator sheet for the construction of the detector paddles will allow separating the gammas and the muons. This feature will require an adjustable voltage level of the triggering threshold of the electronics system. Figure 2 shows the schematics of the BLCRD electronics board. This threshold adjustment can be achieved by substituting R7 and R19 in the electronics board of the BLCRD with resistor pots. This will allow the user to control the voltage level at one of the comparator inputs.

\section{Event Counting}

One of the requirements of the muon witness is to be able to take measurements for long periods of time. The BLCRD output consists of three 8 segment LEDS. In the muon witness detector, this interface will be replaced by a microcontroller board with counting and information storage capabilities. The microcontroller board will be pig tailed to the coincidence detection electronics (which will be very similar to those in the BLCRD board, or even a stripped down one). The connection between the microcontroller board and BLCRD board can be made with a single conductive line going from the output of the AND gate (U4C in Figure 2) that links the two paddles output to a serial input port on the microcontroller board.

The counts should be saved periodically in a permanent memory location. A data EEPROM (Electrically Erasable Programmable Read-Only Memory) could play this role in the Muon Witness detector. This type of memory is cheap and widely available among microcontroller features. The amount of memory required would be set by the estimated unassisted operation time for the Muon Witness detector. 


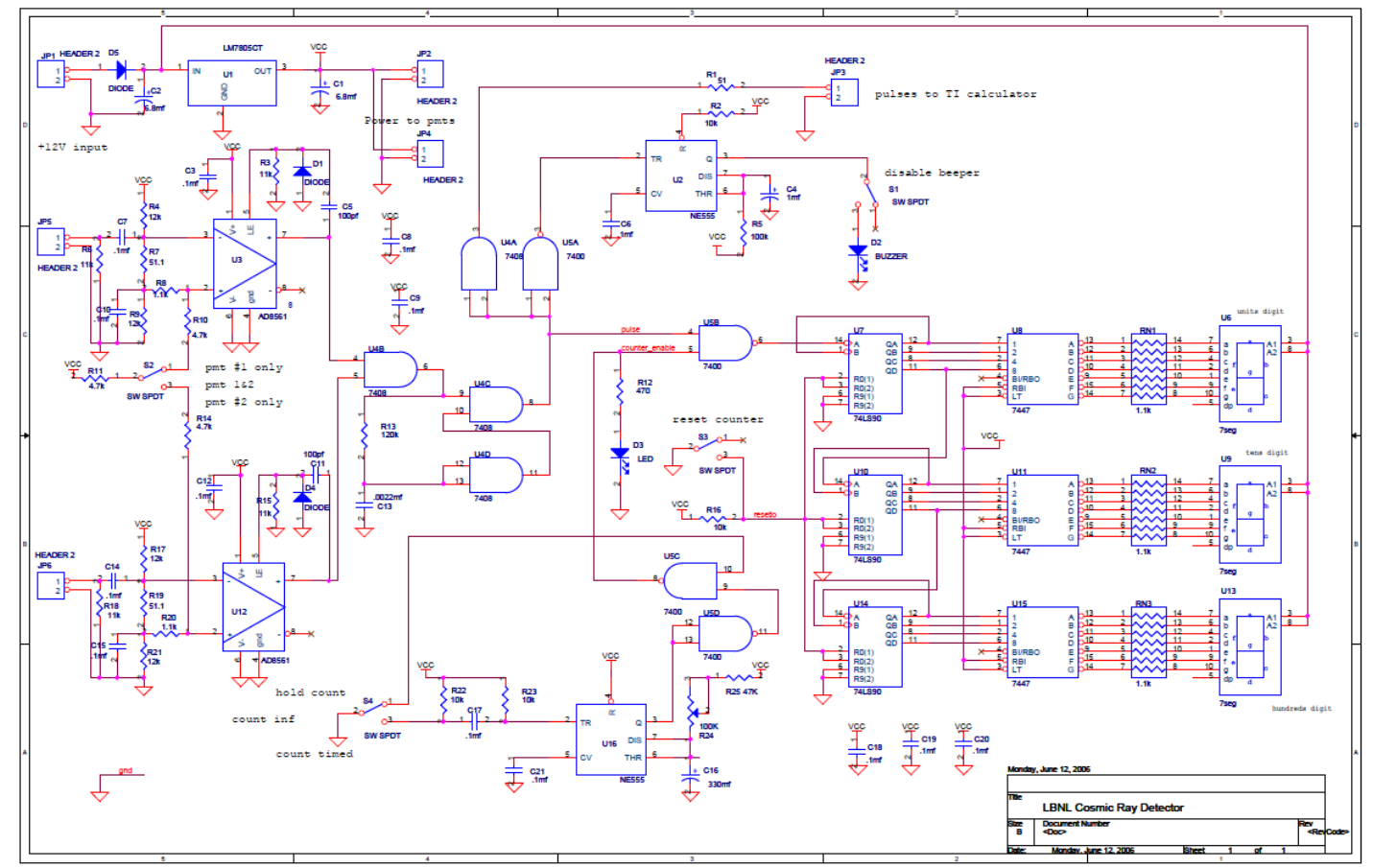

Figure 2: BLCRD electronics board schematics. Taken from [4]

\section{Battery Power Supply}

A lead-cell (car battery) unit featuring concealed spaces is the first candidate battery to power the muon witness. A battery that meets these specifications is the Trojan 12-AGM [12]. Its cost is $\$ 150$. The BLCRD runs at $12 \mathrm{~V}$ and at uses roughly $200-250 \mathrm{~mA}$. At a $20 \mathrm{hr}$ rate (discharge the battery in $20 \mathrm{hrs}$ ) the capacity is rated at $76 \mathrm{AH}$ (amp hours) and at a 5 hour rate the capacity is $67 \mathrm{AH}$. Using the $20 \mathrm{hr}$ rate and a load of $100 \mathrm{~mA}$ (Hrs = AH/Amps), the battery should last about a month (31-32 days). On the assumption that removing the electronics for the display to include the display itself and thereby reducing the load down to 50-60 mA, the charge on the battery should last between 63 and 52 days. This number is a little optimistic as there will be some natural discharge on the battery as it sits and a more realistic estimate would be an operating time of 45-55 days. There is no need for any special electronics to connect up to the battery as the prototype electronics already have voltage regulation built into them.

\begin{tabular}{|l|l|l|}
\hline & BLCRD [3] & Muon Witness (Expected) \\
\hline Plastic detector $(\mathrm{mW})$ & $10 \pm 2$ & $10 \pm 2$ \\
\hline Electronics $(\mathrm{mW})$ & $200 \pm 20$ & $20 \pm 5$ \\
\hline Total $(\mathrm{mW})$ & $220 \pm 25$ & $40 \pm 10$ \\
\hline
\end{tabular}

Table 4: Power budget comparison for the BLCRD and the Muon Witness

\section{Data Read Out}

The PIC-LCD is a simple but powerful board, which uses Microchip's PIC18F8490 Microcontroller [11]. PIC-LCD is equipped with LCD display, three user buttons, LED, possibility for battery power supply, thermistor and buzzer. This board is excellent for applications in monitoring (temperature measuring) and alarm systems. Features of this board include a micro controller unit (PIC18F8490), 16KB Flash memory, 768 B RAM memory, LCD driver, 10 bit ADC, PWM, SPI, I2C, EUSART, TIMERS, COMPARATORS, up to 40MHz and low power $200 \mathrm{kHz}$ operating modes. This board has a sticker price of $\$ 50$ plus the cost of the 
microcontroller programmer. The amount of power used by the microcontroller board will depend on its operation mode, typically the microcontroller will draw a minimum of $2 \mathrm{~mA}$.

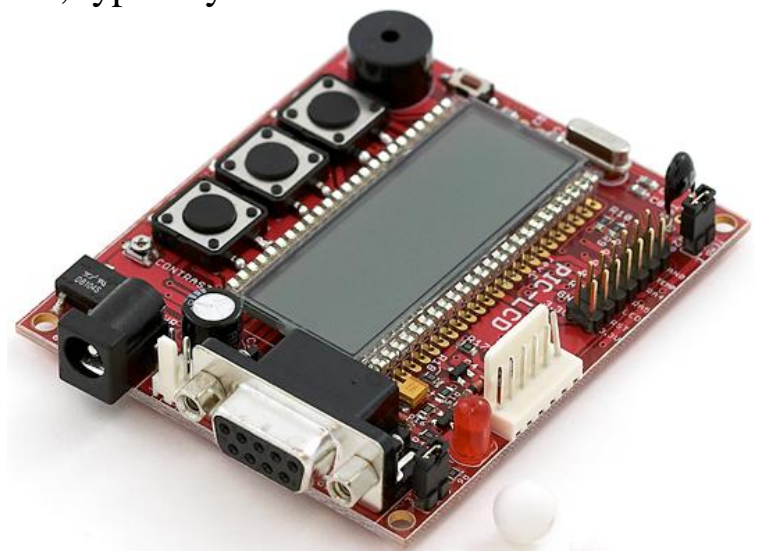

Figure 3: Proposed microcontroller board for the Muon Witness 


\section{Conclusions}

The Berkeley Lab Cosmic Ray Detector assembled by Juniata College was borrowed in December 2010, and has been analyzed as a prototype for a Muon Witness detector. The architecture of this detector will feature a compact, simple and robust way of measuring the muon flux. A detector with these characteristics will be used to estimate activation in ultra low background detector materials. The loaned system was evaluated by operating it in scenarios previously analyzed. In particular, the muon fluence was measured in the underground lab at PNNL and the results show that the prototype has a $30 \%$ measurement error with respect to previous measurements. This error is attributed to gamma ray events. The prototype features a thin scintillator paddle and the muon peak ( $2 \mathrm{MeV}$ for the prototype) and the gamma ray tail (3 $\mathrm{MeV}$ maximum energy) in the energy spectrum are not separated enough to make precise muon flux measurements.

Several design modifications are proposed to build the Muon Witness detector based on the prototype analyzed. Among them, the use of thicker scintillator plastics, addition of a threshold control, and a pig tailed microcontroller board to upgrade the counting electronics. 


\section{References}

[1] Vincente E. Guiseppe for the MAJORANA Collaboration. The MAJORANA Experiment. NOW 2010

[2] Ankeny, A. et al. 2010. "Muon Fluence Measurements for Homeland Security Applications" Pacific Northwest National Laboratory White Paper PNNL- 19632.

[3] Kouzes, R. T. et al. 2009. “Assessment of Cosmic Background Attenuation at Building 3425 (Underground Laboratory)" Pacific Northwest National Laboratory White Paper PNNL18855

[4]Michael Collier and Lyle Wolfley. Assembly Manual for the Berkeley Lab Cosmic Ray Detector

[5] http://pdf.chinaicmart.com/LDS/LDS20361SRA_1229182.pdf

[6] J. F. Ziegler, IBM Journal of Research and Development 42 (1998) 1.

[7] W.N. Hess, H.W. Patterson, R. Wallace, E.L. Chupp, “Cosmic-Ray Neutron Energy Spectrum," Phys. Rev. 116, pp. 445-458 (1959).

[8] Experimental Nuclear Reaction Data (EXFOR) Database Version of December 01, 2010. http://www-nds.iaea.org/exfor/exfor.htm

[9] S. G. Mashnik, “User Manual for the Code CEM95,” JINR, Dubna (1995), OECD NEA Data Bank, Paris, France (1995); RSIC-PSR-357, Oak Ridge, 1995; http://www.nea.fr/abs/html/iaea1247.html.

[10] Knoll G. F. 2002. Radiation Detection and Measurement 3rrd Ed. John Wiley and Sons, New York.

[11] http://www.sparkfun.com/products/773

[12] http://www.trojanbattery.com/Products/24-AGM12V.aspx

[13] Chris Hagmann, David Lange, and Doug Wright. Cosmic-ray Shower Library (CRY) Lawrence Livermore National Laboratory, January 2, 2008 UCRL-TM-229453 